\section{THE ETIOLOGY OF YELLOW FEVER.}

YELLOW fever is an acute infectious disease, endemic in the West Indies, the shores of the Mexican Gulf, and in some parts on the West Coast of Africa, whence the disease has been repeatedly transported into other localities, causing here epidemic outbreaks. Like other infectious diseases, yellow fever is supposed to be caused by a specific living entity which, invading a predisposed person, multiplies there and causes the peculiar pathological changes in the gastrointestinal tract and the liver, characterising yellow fever. Within recent years the supposed specific microbe has been discovered several times. Dr. Domingos Freire, of Brazil, and Dr. Carmona y Valle, of Mexico, have announced such discovery, but Dr. Sternberg, of Wash. ington, who has himself studied the disease on behalf of the United States Government, has shown that none of these discoveries are a reality, and after a prolonged investigation, including the examination of a great many cases affected with, or dead from the disease, has arrived at the following conclusions, embodied in a lengthy report to his Government: that none of the different species of bacilli and cocci, present in the intestinal canal, in the blood, the liver and other tissues of persons affected with yellow fever, can have a claim to be con sidered as the specific microbe; that in a number of cases the examination, microscopic and cultural, of the blood and tissues yielded no bacteria recognisable either by the known methods of staining or culture; and he finally implied that the specific microbe of yellow fever is most probably not of the nature of a bacterium at all. After these very definite conclusions by Dr. Sternberg, it came rather as a surprise when, some month's ago, the announcement was made that Dr. Sanarelli, Professor of Experimental Hygiene in Montevideo, formerly in the Pasteur Institute in Paris, hacl discovered the true cause of yellow fever in the form of a bacillus, Bacillus ictcroides. This surprise is still further heightened by the statement in Dr. Sanarelli's lecture, that the Bacillus icteroides is demonstrable by the ordinary methods of staining and by culture in the ordinary well-known media. The morphological and cultural characters of the bacillus show it to belong to the group of coli-like bacilli; it is rarely demonstrable in a pure state in the blood or tissues, being generally associated with a more or less copious admixture of other microbes-Bacillus coli communis, streptococci and staphylococci; as a rule it is present only in small numbers in the capillary bloodvessels of the liver, spleen and kidney. It reflects great credit on the perseverance and sagacity of Dr. Sanarelli to have been able, notwithstanding all these difficulties, to select out the Bacillus icteroides, and to have by anima experiment been able to demonstrate, at least, as highly probable that the Bacillus icteroides is the true microbe of yellow fever. As mentioned just now, the distribution of the microbe in the affected person, its morphological and cultural characters do not in themselves offer strong prima facie evidence, and Dr. Sanarelli himself fully recognises this; but when we come to the experimental evidence which he furnishes, the evidence as to the Bacillus icteroides being the specific cause of yellow fever assumes considerable power.

In the first place, Sanarelli shows that dogs, goats, and horses are susceptible to infection both with the living bacilli as also and particularly with the highly poisonous toxin produced by the bacilli in broth culture; the symptoms and anatomical lesions hereby produced in these animals in the intestinal tract, the liver and the kidney, bear a striking resemblance to those of yellow fever in man. In the second place, Sanarelli furnishes proof that the toxin produced in broth culture-and separated from the bacillary growth by filtration through a Chamberland filter-when injected in to healthy persons causes a prompt reaction in the form of severe disturbance, primarily of the intestinal tract, but also, further, of the general system closely resembling that in yellow fever. It is to be hoped, nay, it may be assumed as certain, that in continuing his investigations Dr. Sanarelli will ascertain the action of the blood of human beings, who have passed through, and recovered from the disease, on the Bacillus icteroides. This disease, as is well known, very rarely occurs twice in the same person, and it is therefore highly probable that, as is the case in other similar infectious diseases, the blood after a single attack possesses agglutinating action (in vitro), or germicidal action (in corpore), or both on the culture of the specific microbe. If on further investigation the blood serum, after an attack of yellow fever, should be found to show such positive actions on the Bacillus icteroides, a strong support will thereby be furnished as to this bacillus being the specific microbe. It will be the crowning of prolonged and laborious studies, if Sanarelli by experiments on immunisation of animals - the horse being evidently weli fitted for such immunisation-did, as is highly probable that he will, obtain antitoxic serum by which yellow fever can be successfully combated both prophylactically and therapeutically.

E. KLEIN.

\section{THE VARIABLE STAR $\eta$ AQUILE.}

THIS essay contains a full discussion of all the available observations of a remarkable variable star, the fluctuation of whose light presents many features the explanation of which is still beset with much difficulty. Of the 12,000 recorded comparisons of the relative brightness of $\eta$ Aquilie and neighbouring stars, no less than 7147 are due to the indefatigable perseverance of Julius Schmidt, who for the last twenty years of his life (1859-1879) was director of the observatory at Athens. Taking advantage of the preponderance of Schmidt's records over those of all other observers, the author first investigated the variations of the star's light as shown by these alone, and then examined in how far the results thus obtained were confirmed by the labours of other observers. The discussion of Schmidt's observations was complicated by the circumstance that in the majority of cases he only used two comparison stars, $\beta$ and . Aquilæ, one of which there was great reason to believe was itself variable. From the I700 occasions on which both stars were used, Dr. Lockyer found $\imath$ to vary $I^{\prime} 7$ grades of Schmidt.'s scale with a period of about thirty-five years. Associated with this long-period change, the relative brightness of was shown to be subject to an annual variability of about one grade, which is clearly traced to the influence of the hour angle at which the stars were compared. This apparent annual variation is, therefore, identical with the "position error" noted by many observers of variable stars, and which has lately been investigated so thoroughly by $\mathrm{Mr}$. Roberts, of Lovedale, South Africa. Schmidt's observations, freed from both the above variations as well as from the slight aberration error due to the changing position of the earth in its orbit, were all reduced to the meridian of Bonn. Owing to the general sparseness of the data, it was necessary to combine the observations of Ioo periods, or about two years, into a group represented by a single curve. This was effected with the help of a provisional mean period and date of minimum for which Argelander's values of 7 d. $4^{\circ} 23444$ h. and I 848 , mag. I 8 d. 6*4333h. M.T. at Bonn were adopted, the date being that of epoch 400. To further facilitate the construction of the curve the mean

1 "Resultate aus den Beobachtungen des Veränderlichen Sternes n Aquilæ." Inaugural Dissertation zur Erlangung der Doctorwürde, vo William J. S. Lockyer. 5 plates, quarto, pp. 95. (Göttingen: 1897 Londen : Dulau and Co.)

NO. I 446 , VOL. 56$]$ 